\title{
Direct Democracy
}

\section{A Twentieth Century Fund Book}

The Twentieth Century Fund is a research foundation undertaking timely analyses of economic, political, and social issues. Not-for-profit and nompartisan, the Fund was founded in 1919 and endowed by Edward A. Filene.

Board of Trustees of the Twentieth Century Fund

Morris B. Abram

H. Brandt Ayers

Peter A. A. Berle

José A. Cabranes

Joseph A. Califano, Jr.

Alexander Morgan Capron

Edward E. David, Jr.

Brewster C. Denny, Chairman

Charles V. Hamilton

August Heckscher, Emeritus

Matina S. Horner

James A. Leach

Georges-Henri Martin,

Emeritus
Lawrence K. Miller, Emeritus

P. Michael Pitfield

Don K. Price, Emeritus

Richard Ravitch

Arthur M. Schlesinger, Jr., Emeritus

Albert Shanker

Harvey I. Sloane, M.D.

Theodore C. Sorensen

James Tobin, Emeritus

David B. Truman, Emeritus

Shirley Williams

Marcia Bystryn, Acting Director 



\title{
Direct Democracy
}

\author{
The Politics of Initiative, \\ Referendum, and Recall
}

\section{Thomas E. Cronin}

A Twentieth Century Fund Book

HaRvard University Press

Cambridge, Massachusetts, and London, England 1989 
Copyright (C) 1989 by the Twentieth Century Fund, Inc. All rights reserved

Printed in the United States of America

10987654321

This book is printed on acid-free paper, and its binding materials have been chosen for strength and durability.

\section{Library of Congress Cataloging-in-Publication Data}

Cronin, Thomas E.

Direct democracy : the politics of initiative, referendum, and recall / Thomas E. Cronin.

p. $\mathrm{cm}$.

" A Twentieth Century Fund book."

Bibliography: p.

Includes index.

ISBN 0-674-21025-5 (alk. paper)

1. Referendum-United States-History-20th century. 2. Recall-United States-History-20th century. 3. Populism-United States-History-20th century. I. Title.

JF493.U6C76 $1989 \quad 88-14859$

$328^{\prime} .2-\mathrm{dc19}$ CIP 